\title{
Phase relationship between polar faculae and sunspot numbers
}

\author{
L. H. Deng ${ }^{1,2}$ \\ ${ }^{1}$ National Astronomical Observatories/Yunnan Observatory, Chinese Academy of Sciences, \\ 650011, Kunming, China; email: bench@ynao.ac.cn \\ ${ }^{2}$ University of Chinese Academy of Sciences, 100049, Beijing, China
}

\begin{abstract}
Linear and nonlinear approaches are used to investigate the phase asynchrony between the polar faculae (PF) and sunspot numbers (SNs). It is found that, (1) PF show leads of 52 months relative to SNs on the average of the considered time interval; (2) the crosswavelet transform analysis shows that they are asynchronous in both the low- and high-frequency components. These indicate that their phase relationship is not only time-dependent but also frequency-dependent.
\end{abstract}

Keywords. Sun: sunspot - faculae — methods: data analysis

\section{Introduction}

Polar faculae (PF), considered as the main index of high-latitude solar activity, are in anti-phase with sunspot numbers (SNs) (Sheeley 1991, 2008; Makarov \& Makarova 1996; Li et al. 2002; Deng et al. 2011). As PF and SNs represent poloidal magnetic field and toroidal magnetic field respectively, their phase relation is of interest by means of different points of view (Hagino et al. 2004; Callebaut \& Makarova 2008), but it is still poorly investigated. The aim of this poster is to study the phase asynchrony between these two activity indicators at different time scales.
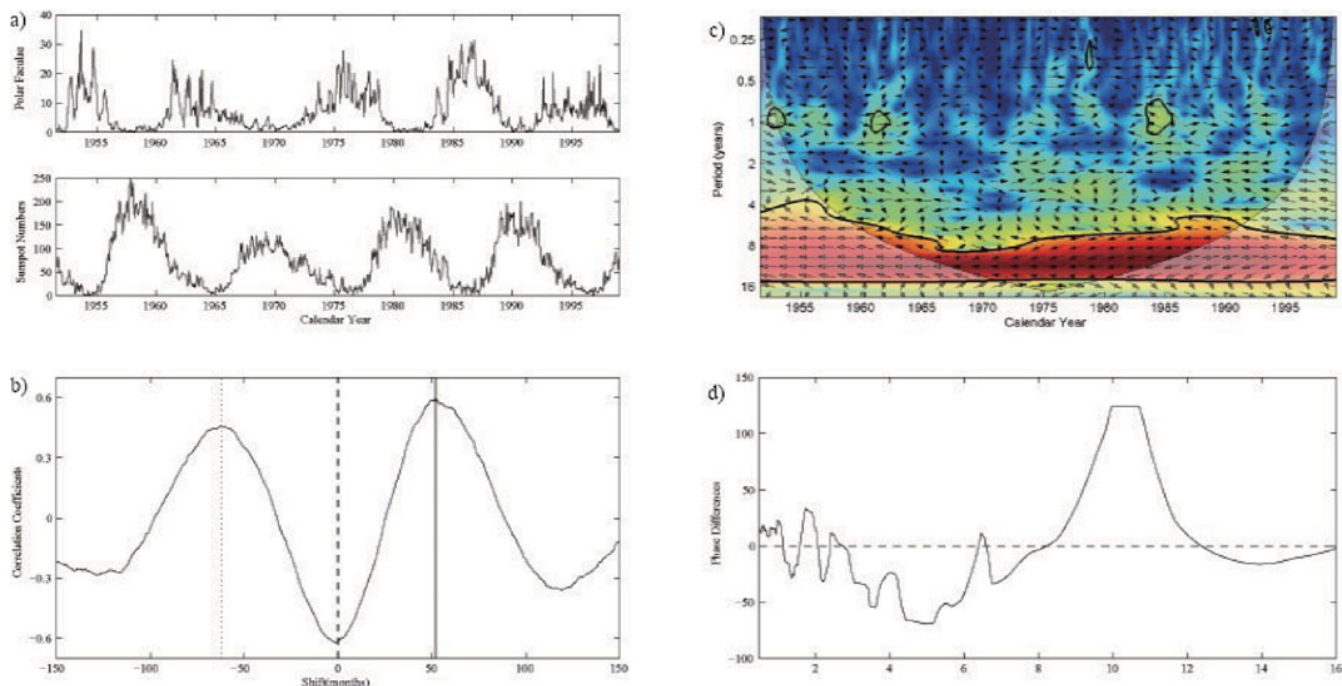

Figure 1. (a) Monthly counts of PF and SNs for the time interval of 1951 August to 1998 December; (b) Correlation coefficients between PF and SNs as a function of shifts; (c) XWT of $\mathrm{PF}$ with respect to SNs; (d) Phase differences between PF and SNs as a function of time scales. 


\section{Data, methods and results}

Monthly counts of PF and SNs are shown in Figure 1(a). They are downloaded from NAOJ and NGDC's web site, respectively. The period of both is from 1951 August to 1998 December. Figure 1(b) shows the result of the cross-correlation coefficients between $\mathrm{PF}$ and SNs. The largest positive correlation with a correlation coefficient of 0.598 occurs when the former has a forward shift of 52 month with respect to the latter. When PF are shifted backward by 62 months, the second largest coefficient of 0.46 is obtained. Therefore, the correlation of $\mathrm{PF}$ is slightly larger with the subsequent sunspot cycle than with the preceding cycle.

Wavelet transform is a powerful tool for analyzing non-stationary signals. Its extension, the so-called cross-wavelet transform (XWT), can be used to the relative phase relation between two time series (Torrence \& Compo 1998). Figure 1(c) shows the XWT of these two activity indictors. The relative phase relation is shown by arrows. Arrows point to the right when the two series are in phase and to the left when they are in anti-phase. Furthermore, we calculate their relative phase differences varying with time scales, which is shown in Figure 1(d). For the time scales of about 8 to 12.5 years, their relative phase differences are positive, indicating that PF lead SNs. However, for both the time scales less than 8 years and larger than 12.5 years, there are no regular oscillatory patterns because their phase differences acutely fluctuate. That is to say, for both the low- and high-frequency components of the XWT, the two are asynchronous.

\section{Conclusion and Discussions}

There are two reasons which mathematically cause phase asynchrony between these two solar activities: (1) PF begin earlier than SNs on the average of the considered time interval; (2) both the low- and high-frequency components of the XWT show strong asynchronous behaviour.

Sheeley $(1991,2008)$ found that polar faculae are well correlated with the polar magnetic field. The polar magnetic field can be understood as the net field transported to the polar zones from the sunspot activity belts by surface flux transport (Cameron et al. 2010) and forms from the sources both in the preceding and the current activity cycle (Tlatov 2007, 2009). PF are also closely related with both the large- and small-scale magnetic field structures (Erofeev \& Erofeeva 2000; Makarov \& Makarova 1996). The origin of solar magnetic field at high latitudes is very complex, we should pay more attention to the solar activity at polar zones in future.

\section{References}

Callebaut, D. K. \& Makarova, V. V. 2008, J. Astrophys. Astr., 29, 69

Cameron, R. H., Jiang, J., Schmitt, D., \& Schussler, M. 2010, ApJ, 719, 264

Deng, L. H., Song, J. Y., Xiang, Y. Y., \& Tang, Y. K. 2011, J. Astrophys. Astr., 32, 401

Erofeev, D. V. \& Erofeeva, A. V. 2000, Sol. Phys., 191, 281

Hagino, M., Sakurai, K., \& Miyazawa, A. 2004, ASP. Conf. Ser., 325, 157

Li, K. J., Irie, M., Wang, J. X., Xiong, S. Y., Yun, H. S., Liang, H. F., Zhan, L. S., \& Zhao, H. J. 2002, Publ. Astron. Soc. Japan, 54, 787

Makarov, V. I. \& Makarova, V. V. 1996, Sol. Phys., 163, 267

Sheeley, N. R., Jr. 1991, ApJ, 374, 386.

Sheeley, N. R., Jr. 2008, ApJ, 680, 1553.

Tlatov, A. G. 2007, Astron. Lett., 33, 771

Tlatov, A. G. 2009, Sol. Phys., 260, 465

Torrence, C. \& Compo, G. P. 1998, Bul. Am. Metero. Soc., 79, 61 